\title{
JOP's counting function and Jones' square function
}

\author{
by
}

KARIN REINHOLD (Albany, NY)

\begin{abstract}
We study a class of square functions in a general framework with applications to a variety of situations: samples along subsequences, averages of $\mathbb{Z}_{+}^{d}$ actions and of positive $L^{1}$ contractions. We also study the relationship between a counting function first introduced by Jamison, Orey and Pruitt, in a variety of situations, and the corresponding ergodic averages. We show that the maximal counting function is not dominated by the square functions.
\end{abstract}

1. Introduction. In [8], Roger Jones introduced the square function for ergodic averages together with the following result.

THEOREM 1.1 (Jones). Let $\tau$ be a measure preserving transformation on a probability space $(X, \beta, m)$. Let $1<p<\infty$. Then, for any sequence $\left\{n_{k}\right\} \subset \mathbb{N}$, the operator

$$
S_{p} f(x)=\left(\sum_{k=1}^{\infty} \frac{\left|f\left(\tau^{n_{k}} x\right)\right|^{p}}{k^{p}}\right)^{1 / p}
$$

is finite a.e. and is of weak type $(1,1)$.

Jones proved Theorem 1.1 for $p=2$, but his technique works for any $p>1$.

In Section 3 we examine what is behind this theorem and extend it to a much larger framework, which can then be applied to a variety of different situations.

Another operator we study is the maximal function associated with a counting function. In [7], B. Jamison, S. Orey and W. Pruitt realized that what was behind the convergence of weighted independent random variables was the behavior of the following counting function. Let $\left\{X_{i}\right\}$ be independent identically distributed random variables and $\left\{w_{i}\right\}$ a sequence of positive numbers. Let $S_{n}=\sum_{i=1}^{n} w_{i} X_{i}$ and $W_{n}=\sum_{i=1}^{n} w_{i}$. Define $N(x)=\left|\left\{n: W_{n} / w_{n} \leq x\right\}\right|$. Jamison, Orey and Pruitt gave conditions

2000 Mathematics Subject Classification: Primary 42B25; Secondary 40A30.

Key words and phrases: square function, counting function, restricted weak type, ergodic averages along subsequences, averages of $\mathbb{Z}_{+}^{d}$ actions and of positive $L^{1}$ contractions. 
on the counting function $N$ necessary to obtain strong laws for the averages $S_{n} / W_{n}$. Later I. Assani in [4] applied this technique to ergodic averages and to sums of independent random variables with weights given by return time sequences.

In Section 4 we examine the counting function associated to averages of operators induced by positive contractions. When these operators are actually induced by measure preserving transformations, we show the relationships between the maximal function associated to the counting functions and the maximal function of the corresponding ergodic averages. Otherwise, we show a domination of a maximal function corresponding to the counting functions by the appropriate square function.

In the measure preserving case, one of the relations between the above mentioned maximal functions is related to extending a restricted weak type inequality to a weak type inequality. Therefore, before we introduce such maximal operators, we discuss what is known about such extensions in Section 2.

Lastly, in Section 5, we apply the results of the previous two sections to averages along subsequences, averages of $\mathbb{Z}_{+}^{d}$ actions, and averages of positive $L^{1}$ contractions. We answer the question of whether the counting function is dominated by the square function in the negative.

As in the definition of the counting function $N(x)$ above, we use henceforth the notation $|A|$ for the cardinality of the set $A$.

2. Restricted weak type. Let $(R, \mu)$ be a $\sigma$-finite measure space and $f$ a measurable function on it. Define $f^{*}$ to be the rearrangement function of $f$,

$$
f^{*}(t)=\sup \{\lambda: \mu(|f|>\lambda)>t\} .
$$

The Lorentz space $L^{p, q}$ consists of all measurable functions, up to measure zero, for which

$$
\|f\|_{p, q}= \begin{cases}\left\{\int_{0}^{\infty}\left[t^{1 / p} f^{*}(t)\right]^{q} \frac{d t}{t}\right\}^{1 / q} & \text { if } 0<q<\infty, \\ \sup _{0<t<\infty}\left\{t^{1 / p} f^{*}(t)\right\} & \text { if } q=\infty\end{cases}
$$

is finite.

The space $L^{p, p}$ coincides with the Lebesgue space $L^{p}$, and $\|f\|_{p, p}=\|f\|_{p}$.

Definitions 2.1. Let $(R, \mu)$ and $(S, \nu)$ be two $\sigma$-finite measure spaces. Suppose $1 \leq p, q \leq \infty$.

(1) $L$ is a weak type $(p, q)$ operator if it is a bounded operator from $L^{p, 1}$ to $L^{q, \infty}$. That is, there exists a constant $C$ such that

$$
\|L f\|_{q, \infty} \leq C\|f\|_{p, 1} \text {. }
$$


(2) $L$ is a uniformly weak type $(p, q)$ operator if it is a bounded operator from $L^{p}$ to $L^{q, \infty}$. That is, there exists a constant $C$ such that

$$
\|L f\|_{q, \infty} \leq C\|f\|_{p} .
$$

(3) $L$ is a strong type $(p, q)$ operator if it is a bounded operator from $L^{p}$ to $L^{q}$. That is, there exists a constant $C$ such that

$$
\|L f\|_{q} \leq C\|f\|_{p}
$$

Notice that weak type $(1,1)$ and uniformly weak type $(1,1)$ coincide, but since $\|f\|_{p} \leq\|f\|_{p, 1}$, uniformly weak type is a stronger condition than weak type for $p>1$. The following is an even weaker condition than weak type.

Definition 2.2. Let $(R, \mu)$ and $(S, \nu)$ be two $\sigma$-finite measure spaces. Suppose $1 \leq p<\infty$ and $1<q \leq \infty$. Let $L$ be an operator defined on the $\mu$-simple functions on $R$ and taking values in the $\nu$-measurable functions on $S$. Then $L$ is of restricted weak type $(p, q)$ if there is a constant $C$ such that

$$
\sup _{t>0} t^{1 / q}\left(L \chi_{E}\right)^{*}(t) \leq C \mu(E)^{1 / p}
$$

for all measurable sets $E \subset R$.

When $q<\infty,(2.1)$ can be reformulated as

$$
\nu\left(\left|L \chi_{E}\right|>\lambda\right) \leq\left(\frac{C}{\lambda} \mu(E)^{1 / p}\right)^{q} \quad \text { for any } \lambda>0 .
$$

The natural question is when restricted weak type $(p, q)$ implies uniformly weak type $(p, q)$. The following theorem from Bennett and Sharpley [5] shows that restricted weak type $(p, q)$ implies weak type $(p, q)$ if $q>1$. In the case of restricted weak type $(1,1)$, Theorem 2.10 below shows that it does not always extend to weak type $(1,1)$.

TheOrem 2.3. Suppose $1 \leq p<\infty$ and $1<q \leq \infty$. Let $L$ be a linear (respectively, nonnegative sublinear) operator defined on the simple functions and suppose $L$ is of restricted weak type $(p, q)$. Then $L$ has a unique extension to a linear (respectively, nonnegative sublinear) operator of weak type $(p, q)$.

Note 2.4. When $p=1$ and $1<q$, this theorem extends to operators $L$ satisfying (a) $L$ is continuous in measure, (b) $L(f+g) \leq L(f)+L(g)$ if the supports of $f$ and $g$ are disjoint, and $L(a f)=|a| L f$.

As a consequence of this theorem, we obtain uniformly weak type for finite measure spaces. 
Corollary 2.5. Let $(R, \mu)$ be a probability space. Suppose $1 \leq p<\infty$ and $1<q \leq \infty$ and let $L$ be a linear (respectively, nonnegative sublinear) operator defined on the simple functions. If $L$ is of restricted weak type $(p, q)$ then $L$ has a unique extension to a linear (respectively, nonnegative sublinear) operator of uniformly weak type $(r, q)$ for any $r>p$.

Proof. This corollary follows straightforwardly from Theorem 2.3 and the following observation about the norm. For any $1 \leq r \leq \infty$, let $r^{\prime}$ denote the conjugate index of $r$, that is, $1 / r+1 / r^{\prime}=1$. Now assume $r>p$.

By definition, since $\mu(|f|>\lambda) \leq \mu(R)=1$, we have $f^{*}(t)=0$ if $t>1$. Thus

$$
\begin{aligned}
\|f\|_{p, 1} & =\int_{0}^{\infty} \frac{f^{*}(t)}{t^{1 / p^{\prime}}} d t=\int_{0}^{1} \frac{f^{*}(t)}{t^{1 / p^{\prime}}} d t \\
& \leq\left[\int_{0}^{1} f^{*}(t)^{r} d t\right]^{1 / r}\left[\int_{0}^{1} \frac{1}{t^{r^{\prime} / p^{\prime}}} d t\right]^{1 / r^{\prime}} \\
& =\|f\|_{r}\left(\frac{p^{\prime}}{p^{\prime}-r^{\prime}}\right)^{1 / r^{\prime}}=\|f\|_{r}\left(\frac{p(r-1)}{r-p}\right)^{(r-1) / r},
\end{aligned}
$$

because $r^{\prime}<p^{\prime}$ as $r>p$. Then, by Theorem 2.3,

$$
\|L f\|_{q, \infty} \leq C(p, r)\|f\|_{r} .
$$

The following interpolation theorem for restricted weak type operators is a consequence of the Marcinkiewicz interpolation theorem. Both can be found in [5].

Theorem 2.6 (E. M. Stein \& G. Weiss). Suppose $1 \leq p_{0}<p_{1}<\infty$ and $1 \leq q_{0}, q_{1} \leq \infty$ with $q_{0} \neq q_{1}$. Let $p$ and $q$ be defined by $1 / p=$ $(1-\theta) / p_{0}+\theta / p_{1}$ and $1 / q=(1-\theta) / q_{0}+\theta / q_{1}$ for some $0<\theta<1$. Let $L$ be a linear (respectively, nonnegative sublinear) operator defined on the simple functions, and suppose that $L$ is of restricted weak types $\left(p_{0}, q_{0}\right)$ and $\left(p_{1}, q_{1}\right)$. If $1 \leq r \leq \infty$, then $L$ has a unique extension to a linear (respectively, nonnegative sublinear) operator, again denoted by $L$, which is bounded from $L^{p, r}$ into $L^{q, r}$. If in addition $p_{j} \leq q_{j}(j=0,1)$, then $L$ is of strong type $(p, q)$.

Curiously, the above theorem does not take care of the case $p_{1}=\infty$, whereas the interpolation theorem of Marcinkiewicz does. But the interpolation with $L^{\infty}$ is easily obtained.

COROLlary 2.7. Let $L$ be a linear (respectively, nonnegative sublinear) operator defined on the simple functions. If $L$ is of restricted weak type $(p, p)$ and satisfies $\left|L \chi_{A}\right| \leq 1$ for any measurable set $A$, then $L$ is of strong type $(q, q)$ for any $q>p$. 
Proof. Since $L$ is of restricted weak type $(p, p)$, it is also of restricted weak type $(q, q)$ for any $q>p$. Indeed, if $0<\lambda<1$,

$$
\nu\left(x:\left|L \chi_{E}\right|>\lambda\right) \leq C \frac{\mu(E)}{\lambda^{p}} \leq C \frac{\mu(E)}{\lambda^{q}},
$$

and if $\lambda \geq 1$,

$$
\nu\left(x:\left|L \chi_{E}\right|>\lambda\right)=0 \leq C \frac{\mu(E)}{\lambda^{q}} .
$$

For any $p<r<q$, there is $0<\theta<1$ such that

$$
\frac{1}{r}=\frac{1-\theta}{p}+\frac{\theta}{q} \text {. }
$$

Since the operator $L$ is of restricted weak types $(p, p)$ and $(q, q)$, Theorem 2.6 asserts that $L$ is of strong type $(r, r)$.

The only satisfactory extensions from restricted weak type to uniformly weak type are Moon's Theorem [13] for convolution operators, and Ash's Theorem [3] for operators that commute with translations.

TheOREM 2.8 (Moon's Theorem). Let $\left\{h_{n}\right\}_{n \geq 1}$ be a sequence of integrable functions on $\mathbb{R}^{d}\left(\right.$ or $\left.\mathbb{T}^{d}\right)$. Let $L f=\sup _{n}\left|f * h_{n}\right|$. Then $L$ is of restricted weak type $(1,1)$ if and only if $L$ is a uniformly weak type $(1,1)$ operator, that is, there is a constant $C>0$ such that

$$
m(x: L f>\lambda) \leq C \frac{\|f\|_{1}}{\lambda},
$$

where $m$ is the Lebesgue measure.

Theorem 2.9 (Ash's Theorem). Let $X=\mathbb{R}$ or $\mathbb{T}=\mathbb{R} / \mathbb{Z}$, with Lebesgue measure. Let $T$ be an operator defined on $L^{2}$. If $T$ commutes with translations, then the following are equivalent:

(1) $T$ is of strong type $(2,2)$,

(2) $T$ is of restricted weak type $(2,2)$.

However, for discrete systems, Akcoglu, Baxter, Bellow and Jones [1] showed that the extension may fail. The next theorem considers convolution operators on $l^{1}(\mathbb{Z})$, where $\mathbb{Z}$ is equipped with counting measure.

THEOREM 2.10. There exists a countable set $\mathcal{C}$ of probability densities $\varphi \in l^{1}(\mathbb{Z})$ such that

$$
M f=\sup _{\varphi \in \mathcal{C}} \varphi * f
$$

is of restricted weak type $(1,1)$ but not of weak type $(1,1)$. 
3. Jones' square function. The main theorem of this section gives a necessary condition for a certain type of "square function" to be a weak $(1,1)$ operator. Square functions were introduced in ergodic theory by Roger Jones when he proved Theorem 1.1 in [8]. The next theorem was inspired by his work.

Let $(X, \beta)$ be a set and a $\sigma$-algebra. Let $m, \nu$ be two positive Borel measures that are $\sigma$-finite on that space. Let $\Gamma$ be a countable infinite set, and $\left\{T_{n}\right\}_{n \in \Gamma}$ a collection of operators defined on the measurable functions on $X$.

Definition 3.1. A collection $\left\{g_{n}\right\}_{n \in \Gamma}$ of nonnegative measurable functions is adapted to the operators $\left\{T_{n}\right\}_{n \in \Gamma}$ with respect to the pair of measures $(m, \nu)$ if there exists a constant $M>0$ such that

$$
\sum_{n \in \Gamma} \nu\left(\left|T_{n} f(x)\right| \geq \lambda g_{n}(x)\right) \leq \frac{M}{\lambda} \int|f| d m
$$

for all $\lambda>0$ and $f \in L^{1}(m)$.

EXAmple 3.2. Let $(X, \beta, m)$ be a probability space, and $\tau: X \rightarrow X$ a measure preserving point transformation. Let $\Gamma=\mathbb{N}$. Given any sequence $\left\{s_{n}\right\} \subset \mathbb{N}$, let $T_{n} f(x)=f\left(\tau^{s_{n}} x\right)$. Let $g_{n}=n$. Then the integrability of functions gives that $\left\{g_{n}\right\}$ is adapted to the operators $\left\{T_{n}\right\}$ with respect to the pair $(m, m)$. Indeed, if $f \in L^{1}(X)$,

$$
\begin{aligned}
\sum_{n=1}^{\infty} m\left(\left|T_{n} f(x)\right| \geq \lambda g_{n}(x)\right) & =\sum_{n=1}^{\infty} m\left(\left|f\left(\tau^{s_{n}} x\right)\right| \geq \lambda n\right) \\
& =\sum_{n=1}^{\infty} m(|f| \geq \lambda n) \leq \int \frac{|f|}{\lambda} d m .
\end{aligned}
$$

Definition 3.3. An operator $T$ is said to be of weak type $\left(L^{1}(m), L^{1}(\nu)\right)$ if there exists a positive constant $C$ such that for any $\lambda>0$, and any $f \in L^{1}(m)$,

$$
\nu(x:|T f(x)|>\lambda) \leq \frac{C}{\lambda} \int|f| d m .
$$

For a nondecreasing function $\Phi:[0, \infty) \rightarrow[0, \infty)$, its inverse is defined by $\Phi^{-1}(u)=\inf \{t: \Phi(t) \geq u\}$. Such a function defines a "square" operator as follows. Let $\left\{T_{n}\right\}_{n \in \Gamma}$ be a sequence of linear operators and $\left\{g_{n}\right\}_{n \in \Gamma}$ a sequence of functions adapted to it with respect to the measures $(m, \nu)$. Define, for integrable functions on $(X, \beta, m)$, the operator

$$
S_{\Phi} f(x)=\Phi^{-1}\left(\sum_{n \in \Gamma} \Phi\left(\frac{\left|T_{n} f(x)\right|}{g_{n}(x)}\right)\right) .
$$


We are now ready to prove a general version of Theorem 1.1 for such a general class of "square" operators.

THEOREM 3.4. Let $\left\{T_{n}\right\}_{n \in \Gamma}$ be a sequence of linear operators, $\left\{g_{n}\right\}_{n \in \Gamma}$ a sequence of functions adapted to it with respect to the measures $(m, \nu)$, $\Phi:[0, \infty) \rightarrow[0, \infty)$ a nondecreasing function, and $S_{\Phi} f$ the corresponding operator as defined above. Let $\beta(\lambda)=\int_{1 /(4 \lambda)}^{\infty} \Phi(1 / t) d t$.

(a) If there exists $\lambda$ such that $\beta(\lambda)<\infty$, then $S_{\Phi} f(x)<\infty \nu$-a.e. for all $f \in L^{1}(m)$.

(b) If in addition $\lim _{\lambda \rightarrow \infty} \beta(\lambda) / \Phi(\lambda)=0$, then $S_{\Phi} f$ satisfies a pseudoweak $(1,1)$ inequality, that is, there exists a function $C(\lambda)$ with $\lim _{\lambda \rightarrow \infty} C(\lambda)=0$ such that

$$
\nu\left(S_{\Phi} f \geq \lambda\right) \leq C(\lambda) \int|f| d m
$$

for all $\lambda>0$ and $f \in L^{1}(m)$. If the operator $S_{\Phi}$ is homogeneous of degree one, $S_{\Phi}(a f)=|a| S_{\Phi} f$, then $S_{\Phi}$ is a weak type $\left(L^{1}(m), L^{1}(\nu)\right)$ operator.

(c) If there exists a constant $K$ such that for all $\lambda>0$,

$$
\lambda \beta(\lambda) \leq K \Phi(\lambda)
$$

then $S_{\Phi} f$ is a weak type $\left(L^{1}(m), L^{1}(\nu)\right)$ operator.

Proof. Let $\lambda>0$. For each $x \in X$, split the index set $\Gamma$ into two pieces: $\Gamma_{1}=\Gamma_{1}(x, \lambda)=\left\{n \in \Gamma:\left|T_{n} f(x)\right| \leq \lambda g_{n}(x)\right\}$ and $\Gamma_{2}=\Gamma_{2}(x, \lambda)=\Gamma \backslash \Gamma_{1}$. Both $\Gamma_{1}$ and $\Gamma_{2}$ depend on $x$ and $\lambda$, but for simplicity we drop their indexing.

(a) Let $\lambda$ be such that $\beta(\lambda)<\infty$. Let $\| t \rrbracket$ denote the integer part of $t \in \mathbb{R}$. By noticing that

$$
\sum_{n \in \Gamma_{1}} \Phi\left(\frac{\left|T_{n} f\right|}{g_{n}}\right)=\sum_{n \in \Gamma} \chi_{\left[\left|T_{n} f\right| \leq \lambda g_{n}\right]} \Phi\left(\frac{\left|T_{n} f\right|}{g_{n}}\right),
$$

we have

$$
\begin{gathered}
\int \sum_{n \in \Gamma_{1}} \Phi\left(\frac{\left|T_{n} f\right|}{g_{n}}\right) d \nu \leq \sum_{n \in \Gamma} \int_{\left[\left|T_{n} f\right| \leq \lambda g_{n}\right]} \Phi\left(\frac{\left|T_{n} f\right|}{g_{n}}\right) d \nu \\
\leq \sum_{n \in \Gamma} \sum_{j \geq \llbracket \log _{2}(1 / \lambda) \llbracket\left[1 / 2^{j+1}<\left|T_{n} f\right| / g_{n} \leq 1 / 2^{j}\right]} \Phi\left(\frac{\left|T_{n} f\right|}{g_{n}}\right) d \nu \\
\leq \sum_{j \geq \rrbracket \log _{2}(1 / \lambda) \rrbracket} \Phi\left(\frac{1}{2^{j}}\right) \sum_{n \in \Gamma} \nu\left(\frac{1}{2^{j+1}}<\frac{\left|T_{n} f\right|}{g_{n}} \leq \frac{1}{2^{j}}\right) \\
\leq M \sum_{j \geq \rrbracket \log _{2}(1 / \lambda) \rrbracket} 2^{j+1} \Phi\left(\frac{1}{2^{j}}\right) \int|f| d m
\end{gathered}
$$




$$
\begin{aligned}
& \leq M \sum_{j \geq \rrbracket \log _{2}(1 / \lambda) \rrbracket} 4 \int_{2^{j-1}}^{2^{j}} \Phi(1 / t) d t \int|f| d m \\
& \leq 4 M \int_{2^{\log _{2}(1 / \lambda)-2}} \Phi(1 / t) d t \int|f| d m \leq 4 M \beta(\lambda) \int|f| d m,
\end{aligned}
$$

where the fourth inequality is due to (3.1).

Since $\beta(\lambda)<\infty$, we see that for any $f \in L^{1}(m)$,

$$
\sum_{n \in \Gamma_{1}} \Phi\left(\frac{\left|T_{n} f\right|}{g_{n}}\right)=\sum_{n \in \Gamma} \chi_{\left[\left|T_{n} f\right| \leq \lambda g_{n}\right]} \Phi\left(\frac{\left|T_{n} f\right|}{g_{n}}\right)<\infty
$$

for $\nu$-almost every $x$.

The sums over $\Gamma_{2}$ are also finite for $\nu$-almost every $x$, for any $f \in L^{1}(m)$, because, by $(3.1), \Gamma_{2}=\Gamma_{2}(x, \lambda)$ is a finite set for $\nu$-almost every $x$. Indeed,

$$
\int\left|\Gamma_{2}\right| d \nu=\sum_{n \in \Gamma} \nu\left(\left|T_{n} f\right|>\lambda g_{n}\right) \leq \frac{M}{\lambda} \int|f| d m .
$$

Combining the sums over $\Gamma_{1}$ and $\Gamma_{2}$ yields $S_{\Phi} f(x)<\infty$ for $\nu$-almost every $x$, for any $f \in L^{1}(m)$.

(b) Since $\Phi$ is increasing and $\Phi(0) \geq 0$, there exists $\lambda_{0}=\inf \{\lambda>0$ : $\Phi(\lambda)>0\}$. If $0<\lambda \leq \lambda_{0}$,

$$
\begin{aligned}
\nu\left(S_{\Phi} f \geq \lambda\right) & \leq \nu\left(S_{\Phi} f>0\right) \leq \sum_{n \in \Gamma} \nu\left(\Phi\left(\frac{\left|T_{n} f\right|}{g_{n}}\right)>0\right) \\
& \leq \sum_{n \in \Gamma} \nu\left(\left|T_{n} f\right| \geq \lambda_{0} g_{n}\right) \leq \frac{M}{\lambda_{0}} \int|f| d m \leq \frac{M}{\lambda} \int|f| d m .
\end{aligned}
$$

If $\lambda>\lambda_{0}$,

$$
\begin{aligned}
\nu\left(S_{\Phi} f \geq \lambda\right) & \leq \nu\left[\sum_{n \in \Gamma} \Phi\left(\frac{\left|T_{n} f\right|}{g_{n}}\right) \geq \Phi(\lambda)\right] \\
& \leq \nu\left[\sum_{n \in \Gamma_{1}} \Phi\left(\frac{\left|T_{n} f\right|}{g_{n}}\right) \geq \frac{\Phi(\lambda)}{2}\right]+\nu\left[\sum_{n \in \Gamma_{2}} \Phi\left(\frac{\left|T_{n} f\right|}{g_{n}}\right) \geq \frac{\Phi(\lambda)}{2}\right] .
\end{aligned}
$$

To handle the first term, we use the estimate obtained in (3.2),

$$
\begin{aligned}
\nu\left[\sum_{n \in \Gamma_{1}} \Phi\left(\frac{\left|T_{n} f\right|}{g_{n}}\right)>\frac{\Phi(\lambda)}{2}\right] & \leq \frac{2}{\Phi(\lambda)} \int \sum_{n \in \Gamma_{1}} \Phi\left(\frac{\left|T_{n} f\right|}{g_{n}}\right) d \nu \\
& \leq 8 M \frac{\beta(\lambda)}{\Phi(\lambda)} \int|f| d m
\end{aligned}
$$


For the other term,

$$
\nu\left[\sum_{n \in \Gamma_{2}} \Phi\left(\frac{\left|T_{n} f\right|}{g_{n}}\right)>\frac{\Phi(\lambda)}{2}\right] \leq \nu\left[\sum_{n \in \Gamma_{2}} \Phi\left(\frac{\left|T_{n} f\right|}{g_{n}}\right)>0\right] .
$$

But

$$
\sum_{n \in \Gamma_{2}} \Phi\left(\frac{\left|T_{n} f\right|}{g_{n}}\right)=\sum_{n \in \Gamma} \chi_{\left[\left|T_{n} f\right|>\lambda g_{n}\right]} \Phi\left(\frac{\left|T_{n} f\right|}{g_{n}}\right) .
$$

Thus

$$
\nu\left[\sum_{n \in \Gamma_{2}} \Phi\left(\frac{\left|T_{n} f\right|}{g_{n}}\right)>0\right] \leq \sum_{n \in \Gamma} \nu\left(\left|T_{n} f\right|>\lambda g_{n}\right) \leq \frac{M}{\lambda} \int|f| d m
$$

Letting

$$
C(\lambda)=8 M \frac{\beta(\lambda)}{\Phi(\lambda)}+\frac{M}{\lambda}
$$

we have proven

$$
\nu\left(S_{\Phi} f>\lambda\right) \leq C(\lambda) \int|f| d m,
$$

where under the assumption of (b), $C(\lambda) \rightarrow 0$ as $\lambda \rightarrow \infty$.

(c) Under the assumption of (c), $\beta(\lambda) / \Phi(\lambda) \leq K / \lambda$. Hence, $C(\lambda) \leq C^{\prime} / \lambda$, proving the desired weak type $\left(L^{1}(m), L^{1}(\nu)\right)$ inequality.

Corollary 3.5. Let $\left\{T_{n}\right\}_{n \in \Gamma}$ and $\left\{g_{n}\right\}_{n \in \Gamma}$ be as in Theorem 3.4. Then

$$
S_{p} f=\left[\sum_{n \in \Gamma}\left(\frac{\left|T_{n} f\right|}{g_{n}}\right)^{p}\right]^{1 / p}
$$

is a weak type $\left(L^{1}(m), L^{1}(\nu)\right)$ operator for any $1<p<\infty$.

Theorem 3.4 has applications to a variety of situations. We will use it for averages of $\mathbb{Z}^{d}$ actions and averages of positive $L^{1}$ contraction operators in Section 5.

4. JOP's counting function. Let $\Gamma$ be a countable set, and $\left\{\Gamma_{i}\right\}_{i=1}^{\infty}$ a nested sequence of finite sets such that $\bigcup_{i=1}^{\infty} \Gamma_{i}=\Gamma$. Let $D_{i}=\Gamma_{i} \backslash \Gamma_{i-1}$, with $\Gamma_{0}=\emptyset$.

Let $(X, \beta)$ be a set endowed with a $\sigma$-algebra, and $\nu, m$ two positive Borel $\sigma$-finite measures on it. Let $\left\{T_{n}\right\}_{n \in \Gamma}$ be a family of linear operators defined on the measurables functions on $(X, \beta)$.

Given a sequence $\left\{g_{k}\right\}_{k \in \Gamma}$ of positive functions, adapted to the family $\left\{T_{k}\right\}$ with respect to $(m, \nu)$, define $G_{n}(x)$, their dominant on each $\Gamma_{n}$, by

$$
G_{n}(x)=\max _{k \in \Gamma_{n}} g_{k}(x) \text {. }
$$

Since the $\Gamma_{n}$ 's are nested, $\left\{G_{n}(x)\right\}_{n}$ is increasing for each $x$. 
Define the partial sums and the averages operators respectively by

$$
S_{n} f=\sum_{k \in \Gamma_{n}} T_{k} f \quad \text { and } \quad B_{n} f=\frac{S_{n} f}{G_{n}},
$$

and, for any given $\alpha>0$, the counting function

$$
N_{\alpha} f(x)=\sum_{n=1}^{\infty}\left|\left\{k \in D_{n}: \frac{\left|T_{k} f(x)\right|}{G_{n}(x)} \geq \frac{1}{\alpha}\right\}\right| .
$$

The corresponding maximal functions for the averages and the counting functions are

$$
B^{*} f=\sup _{1 \leq n<\infty}\left|B_{n} f\right| \quad \text { and } \quad N^{*} f=\sup _{\alpha>0} \frac{N_{\alpha} f}{\alpha}
$$

respectively. The counting functions $N_{\alpha}$ first appeared in Jamison, Orey and Pruitt [7] to study strong laws of weighted averages of independent random variables. Later Assani [4] applied them to ergodic theory.

The following lemmas state the basic properties of these operators.

Lemma 4.1. The operators $N_{\alpha}$ have the following properties:

(a) $N_{\alpha} f(x)$ is increasing in $\alpha$, that is, if $\alpha<\beta$, then $N_{\alpha} f(x) \leq N_{\beta} f(x)$.

(b) $N_{\alpha}($ af $)=N_{|a| \alpha} f$ for any $a \neq 0$.

(c) $N_{\alpha}\left(f_{1}+f_{2}\right) \leq N_{2 \alpha} f_{1}+N_{2 \alpha} f_{2}$ for any pair of measurable functions and any $\alpha>0$.

$$
\int \frac{N_{\alpha} f}{\alpha} d \nu \leq M \int|f| d m
$$

for all $\alpha>0$, where $M$ is the constant in (3.1).

Proof. (a) is immediate by definition of $N_{\alpha}$, and (b) follows by the linearity of the operators $T_{k}$. Property (c) follows from the inequality

$$
\begin{aligned}
N_{\alpha}(f+g)(x)= & \sum_{n=1}^{\infty}\left|\left\{k \in D_{n}: \frac{\left|T_{k} f_{1}(x)+T_{k} f_{2}(x)\right|}{G_{n}(x)}>\frac{1}{\alpha}\right\}\right| \\
\leq & \sum_{n=1}^{\infty}\left|\left\{k \in D_{n}: \frac{\left|T_{k} f_{1}(x)\right|}{G_{n}(x)}>\frac{1}{2 \alpha}\right\}\right| \\
& +\sum_{n=1}^{\infty}\left|\left\{k \in D_{n}: \frac{\left|T_{k} f_{2}(x)\right|}{G_{n}(x)}>\frac{1}{2 \alpha}\right\}\right| \\
= & N_{2 \alpha} f_{1}(x)+N_{2 \alpha} f_{2}(x) .
\end{aligned}
$$


Since $\left\{g_{n}\right\}$ is adapted to $\left\{T_{n}\right\}$ with respect to the measures $(m, \nu)$, inequality (3.1) gives property $(\mathrm{d})$ :

$$
\begin{aligned}
\int \frac{N_{\alpha} f}{\alpha} d \nu & \leq \frac{1}{\alpha} \sum_{n} \sum_{k \in D_{n}} \nu\left(\left|T_{k} f\right| \geq \frac{G_{n}}{\alpha}\right) \\
& \leq \frac{1}{\alpha} \sum_{k \in \Gamma} \nu\left(\left|T_{k} f\right| \geq \frac{g_{k}}{\alpha}\right) \leq M \int|f| d m
\end{aligned}
$$

Lemma 4.2. The operator $N^{*}$ has the following properties:

(a) $N^{*}$ is homogeneous of degree 1 , that is, $N^{*}($ af $)=|a| N^{*} f$ for all scalar $a$.

(b) $N^{*}(f+g) \leq 2\left(N^{*} f+N^{*} g\right)$.

Proof. Property (a) follows from Lemma 4.1(b), and property (b) from Lemma 4.1(c).

For the next lemma, we need to know which $G_{n}$ 's are the same. Define then, for each $x$,

$$
m(n)=m(n, x)=\max \left\{r: G_{r}(x)=G_{n}(x)\right\} .
$$

LEMma 4.3. Let the operators $T_{k}$ be induced by measure preserving transformations $\tau_{k}, T_{k} f(x)=f\left(\tau_{k} x\right)$. Then

(a) for any measurable set $A$,

$$
\frac{N_{G_{m(n)}(x)} \chi_{A}(x)}{G_{m(n)}(x)}=B_{m(n)} \chi_{A}(x) \quad \text { and } \quad N^{*} \chi_{A}=B^{*} \chi_{A}, \quad \nu \text {-a.e.; }
$$

(b) if $\sup _{n}\left|\Gamma_{n}\right| / G_{n} \leq C<\infty \nu$-almost everywhere, then $N^{*}$ is a bounded operator in $L^{\infty}$. Moreover

$$
N^{*} f \leq \sup _{n \geq 1} \frac{\left|\Gamma_{n}\right|}{G_{n}}\|f\|_{\infty} \quad \nu \text {-almost everywhere. }
$$

Proof. Given $\alpha>0$, let $n(\alpha)=n(\alpha, x)=\max \left\{n: G_{n}(x) \leq \alpha\right\}$ (we drop the $x$ to simplify notation).

(a) We have

$$
\begin{aligned}
N_{\alpha} \chi_{A}(x) & =\sum_{n=1}^{\infty}\left|\left\{k \in D_{n}: \frac{T_{k} \chi_{A}(x)}{G_{n}(x)} \geq \frac{1}{\alpha}\right\}\right|=\left|\left\{k \in \Gamma_{n(\alpha)}: \chi_{A}\left(\tau_{k} x\right)=1\right\}\right| \\
& =\sum_{k \in \Gamma_{n(\alpha)}} \chi_{A}\left(\tau_{k} x\right)=S_{n(\alpha)} \chi_{A}(x) .
\end{aligned}
$$

Thus

$$
\frac{N_{\alpha} \chi_{A}}{\alpha}=B_{n(\alpha)} \chi_{A} \frac{G_{n(\alpha)}}{\alpha} \leq B_{n(\alpha)} \chi_{A},
$$


which shows

$$
N^{*} \chi_{A}(x) \leq B^{*} \chi_{A}(x)
$$

On the other hand,

$$
\frac{N_{G_{m(n)}(x)} \chi_{A}(x)}{G_{m(n)}(x)}=B_{m(n)} \chi_{A}(x) \text { and } B^{*} \chi_{A}=\sup _{n} B_{m(n)} \chi_{A} .
$$

Therefore $B^{*} \chi_{A}(x) \leq N^{*} \chi_{A}(x)$. Thus $N^{*} \chi_{A}=B^{*} \chi_{A}$.

(b) Assume $f \in L^{\infty}$. Then

$$
\begin{aligned}
N_{\alpha} f(x) & =\sum_{n=1}^{\infty}\left|\left\{k \in D_{n}: \frac{\left|f\left(\tau_{k} x\right)\right|}{G_{n}(x)} \geq \frac{1}{\alpha}\right\}\right| \\
& \leq \sum_{\left\{n: G_{n}(x) \leq \alpha\|f\|_{\infty}\right\}}\left|D_{n}\right|=\sum_{1 \leq n \leq n\left(\alpha\|f\|_{\infty}\right)}\left|D_{n}\right|=\left|\Gamma_{n\left(\alpha\|f\|_{\infty}\right)}\right| .
\end{aligned}
$$

Thus

$$
\frac{N_{\alpha} f(x)}{\alpha} \leq \frac{\left|\Gamma_{n\left(\alpha\|f\|_{\infty}\right)}\right|}{G_{n\left(\alpha\|f\|_{\infty}\right)}}\|f\|_{\infty} \leq \sup _{n} \frac{\left|\Gamma_{n}\right|}{G_{n}}\|f\|_{\infty} .
$$

The following theorem is the main ingredient of this section. It shows the interrelationship between the maximal operators $N^{*}$ and $B^{*}$. We will apply this result later on to two types of averages with different natures: averages on $\mathbb{Z}^{d}$, and averages along subsequences (in $\mathbb{Z}$ ). For both we obtain similar results, but the structure needed to handle both cases simultaneously is a little intricate. Bear in mind that, in these applications, $G_{n}=n^{d}$ for some $d \in \mathbb{N}$.

TheOREM 4.4. Let $(X, \beta, m)$ be a probability space. Let $\left\{T_{k}\right\}_{n \in \Gamma}$ be a family of operators induced by measure preserving transformations as above, and let $\left\{g_{k}\right\}_{n \in \Gamma}$ be a sequence of functions adapted to it with respect to the pair $(m, m)$. Assume that $\left\{g_{k}\right\}$ also satisfies the following growth conditions, given in terms of the sequence $\left\{G_{n}\right\}$ defined in (4.1):

(a) there exists a constant $K$ such that $\sup _{n} G_{n+1} / G_{n} \leq K$ a.e.,

(b) there exists a constant $\kappa$ such that $\sup _{n} G_{n}^{s-1} \sum_{j \geq n}\left[G_{j}-G_{j-1}\right] / G_{j}^{s}$ $\leq \kappa$ a.e. for any $1<s<\infty$,

(c) $\sup _{n}\left|\Gamma_{n}\right| / G_{n} \in L^{\infty}$,

(d) $G_{1} \geq 1$.

Let $1 \leq p<\infty$.

(i) If $N^{*}$ is of uniformly weak type $(p, p)$ then $B^{*}$ is of strong type $(r, r)$ for all $r>p$, and if $p>1$ then $B^{*}$ is also of weak type $(p, p)$.

(ii) If $B^{*}$ is of uniformly weak type $(p, p)$ then $N^{*}$ is of strong type $(r, r)$ for all $r>p$. 
Proof. (i) Since by Lemma $4.3, N^{*} \chi_{A}=B^{*} \chi_{A}$, and $N^{*}$ is a uniformly weak type $(p, p)$ operator, $B^{*}$ is of restricted weak type $(p, p)$. Thus, since $B^{*}$ is a nonnegative sublinear operator, by Corollary 2.7, it is of strong type $(r, r)$ for all $r>p$. Now, if $p>1$, then by Theorem $2.3, B^{*}$ is of weak type $(p, p)$ as well.

(ii) Since $B^{*}$ is a uniformly weak type $(p, p)$ operator and $N^{*} \chi_{A}=B^{*} \chi_{A}$, $N^{*}$ is of restricted weak type $(p, p)$. However, $N^{*}$ may fail to be subadditive, and hence we cannot apply the results of Section 2. Instead, this direction follows as in Assani's [4].

First note that by (3.1), if we let

$$
\widetilde{f}(x)=\sup _{n \geq 1} \max _{k \in D_{n}} \frac{\left|T_{k} f(x)\right|}{G_{n}(x)},
$$

then $\tilde{f}$ is finite almost everywhere for any $f \in L^{1}$.

Let $r>p$ and $f \in L^{r}$. Write $r=p s$, with $s>1$. Let

$$
N^{*} f \leq \sup _{0<\alpha \leq 1} \frac{N_{\alpha} f}{\alpha}+\sup _{\alpha \geq 1} \frac{N_{\alpha} f}{\alpha}=N_{1}^{*} f+N_{2}^{*} f .
$$

The first term is simple to handle. Indeed, if $\alpha_{0}=[2 \widetilde{f}(x)]^{-1}$, then $N_{\alpha} f(x)=0$ for all $\alpha<\alpha_{0}$. And since $N_{\alpha} f$ is increasing in $\alpha$,

$$
N_{1}^{*} f(x)=\sup _{\alpha_{0} \leq \alpha \leq 1} \frac{N_{\alpha} f}{\alpha} \leq \frac{N_{1} f(x)}{\alpha_{0}}=2 \widetilde{f}(x) N_{1} f(x) .
$$

Since $f \in L^{r} \subset L^{1},(3.1)$ gives that $N_{1} f$ is finite almost everywhere. Thus $N_{1}^{*} f(x)<\infty$ almost everywhere.

For the second term, observe that by the growth condition (c), and the fact that $\Gamma_{n} \rightarrow \Gamma$ and $\Gamma$ is an infinite set, we have $G_{n}(x) \rightarrow \infty$ for almost every $x$. Then, since $N_{\alpha} f(x)$ is increasing in $\alpha$, and by the growth condition (a),

$$
N_{2}^{*} f(x) \leq K \sup _{n \geq 1} \frac{N_{G_{n}(x)} f(x)}{G_{n}(x)} \quad \text { a.e. }
$$

Now,

$$
N_{G_{n}(x)} f(x) \leq\left|\Gamma_{n}\right|+\sum_{j \geq n}\left|\left\{k \in D_{j}: \frac{\left|T_{k} f(x)\right|}{G_{j}(x)} \geq \frac{1}{G_{n}(x)}\right\}\right|=\left|\Gamma_{n}\right|+R_{n} f(x) .
$$

The last term is estimated by

$$
\begin{aligned}
\frac{R_{n} f}{G_{n}^{s}} & \leq \sum_{j \geq n} \sum_{k \in D_{j}}\left(\frac{\left|T_{k} f\right|}{G_{j}}\right)^{s}=\sum_{j \geq n} \frac{1}{G_{j}^{s}} \sum_{k \in D_{j}} T_{k}\left(|f|^{s}\right) \\
& =\sum_{j \geq n} \frac{1}{G_{j}^{s-1}} \frac{S_{j}\left(|f|^{s}\right)-S_{j-1}\left(|f|^{s}\right)}{G_{j}}
\end{aligned}
$$




$$
\begin{aligned}
= & \sum_{j \geq n} \frac{1}{G_{j}^{s-1}}\left[B_{j}\left(|f|^{s}\right)-B_{j-1}\left(|f|^{s}\right)\right]+\sum_{j \geq n} \frac{G_{j}-G_{j-1}}{G_{j}^{s}} B_{j-1}\left(|f|^{s}\right) \\
\leq & \sum_{j \geq n}\left[\frac{1}{G_{j}^{s-1}}-\frac{1}{G_{j+1}^{s-1}}\right] B_{j}\left(|f|^{s}\right)+B_{n-1}\left(|f|^{s}\right) \frac{1}{G_{n}^{s-1}} \\
& +B^{*}|f|^{s} \sum_{j \geq n} \frac{G_{j}-G_{j-1}}{G_{j}^{s}} \\
\leq & (1+\kappa) B^{*}|f|^{s} \frac{1}{G_{n}^{s-1}},
\end{aligned}
$$

where the last inequality is due to the growth condition (b). Let $L=$ $\left\|\sup _{n}\left|\Gamma_{n}\right| / G_{n}\right\|_{\infty}$, which is finite by condition (c), and put $\kappa^{\prime}=1+\kappa$. Since $G_{1} \geq 1$,

$$
\sup _{n \geq 1} \frac{N_{G_{n}} f}{G_{n}} \leq \sup _{n \geq 1} \frac{\left|\Gamma_{n}\right|}{G_{n}}+\sup _{n \geq 1} \frac{R_{n} f}{G_{n}} \leq L+\kappa^{\prime} B^{*}|f|^{s} .
$$

Since $f \in L^{r}$ and $r=p s$, we have $|f|^{s} \in L^{p}$. By hypothesis, $B^{*}$ is of uniformly weak type $(p, p)$. Therefore $B^{*}|f|^{s}$ is finite a.e., showing that $\sup _{n \geq 1} N_{G_{n}} f / G_{n}$ is also finite almost everywhere.

From (4.2) and (4.3), we have

$$
N^{*} f \leq 2 \widetilde{f} N_{1} f+K\left(L+\kappa^{\prime} B^{*}|f|^{s}\right) .
$$

But then

$$
\begin{aligned}
m\left[N^{*} f \geq 2+K\left(L+\kappa^{\prime}\right)\right] & \leq m\left(\tilde{f} N_{1} f \geq 1\right)+m\left(B^{*}|f|^{s} \geq 1\right) \\
& \leq m(\tilde{f}>1)+m\left(N_{1} f \geq 1\right)+m\left(B^{*}|f|^{s} \geq 1\right) \\
& \leq C_{1} \int_{[|f| \geq 1]}|f| d m+C_{2} \int|f|^{r} d m \leq C_{3} \int|f|^{r} d m .
\end{aligned}
$$

Thus, if $\lambda>0$, by the homogeneity of $N^{*}$,

$$
\begin{aligned}
m\left(N^{*} f \geq \lambda\right) & =m\left[N^{*}\left(\frac{2+K\left(L+\kappa^{\prime}\right)}{\lambda} f\right) \geq 2+K\left(L+\kappa^{\prime}\right)\right] \\
& \leq C_{3}\left[\frac{2+K\left(L+\kappa^{\prime}\right)}{\lambda}\right]^{r}\|f\|_{r}^{r} .
\end{aligned}
$$

The above theorem yields convergence results. The same techniques used in Assani [4] prove the following corollary. Part of this corollary was already obtained by Jones, Rosenblatt and Wierdl [11].

COROLlary 4.5. With the assumptions of Theorem 4.4 and assuming that $\lim _{n \rightarrow \infty} B_{n} \chi_{A}(x)$ exists a.e. for all measurable sets $A$, we have: 
(a) If $N^{*}$ is of uniformly weak type $(p, p)$, then $\lim _{n \rightarrow \infty} N_{G_{n}} f(x) / G_{n}$ exists a.e. for all $f \in L^{p}$ and $\lim _{n \rightarrow \infty} B_{n} f(x)$ exists a.e. for all $f \in L^{r}, r>p$.

(b) If $B^{*}$ is of uniformly weak type $(p, p)$, then $\lim _{n \rightarrow \infty} B_{n} f(x)$ exists a.e. for all $f \in L^{p}$ and $\lim _{n \rightarrow \infty} N_{n} f(x) / n$ exists a.e. for all $f \in L^{r}$, $r>p$.

\section{Applications}

5.1. Counting along a subsequence. Let $(X, \beta, m)$ be a probability space and $\tau: X \rightarrow X$ a measure preserving transformation. Let $n_{k}$ be an increasing sequence of positive integers. Define

$$
B_{n} f(x)=\frac{1}{n} \sum_{k=1}^{n} f\left(\tau^{n_{k}} x\right), \quad N_{\alpha} f(x)=\left|\left\{k \geq 1: \frac{\left|f\left(\tau^{n_{k}} x\right)\right|}{k} \geq \frac{1}{\alpha}\right\}\right| .
$$

Let also

$$
B^{*} f=\sup _{n}\left|B_{n} f\right|, \quad N^{*} f=\sup _{\alpha>0} \frac{N_{\alpha} f}{\alpha} .
$$

COROLlaRY 5.1. Let $n_{k}$ be an increasing sequence of positive integers. Let $1 \leq p<\infty$.

(a) If $N^{*}$ is of uniformly weak type $(p, p)$ then $B^{*}$ is of strong type $(r, r)$ for all $r>p$, and if $p>1$ then $B^{*}$ is also of weak type $(p, p)$.

(b) If $B^{*}$ is of uniformly weak type $(p, p)$ then $N^{*}$ is of strong type $(r, r)$ for all $r>p$.

Proof. If we let $\Gamma=\mathbb{Z}_{+}, \Gamma_{n}=[1, n], T_{k} f(x)=f\left(\tau^{n_{k}} x\right)$, and $g_{k}=k$, all the hypotheses of Theorem 4.4 are satisfied.

Jones, Rosenblatt and Wierdl in [11] showed that if $f \in L^{1}$, then for any $1<p<\infty, S_{p} f=\left(\sum_{k}\left|f\left(\tau^{n_{k}} x\right) / k\right|^{p}\right)^{1 / p}<\infty$ a.e. whenever $N^{*} f<\infty$ a.e. But we already know, by Corollary 3.5, that $S_{p} f<\infty$ a.e. for any $f \in L^{1}$. However, $N^{*} f$ may fail to be finite.

Using Corollary 5.3 below, we will show how to construct an $f \in L^{1}$ such that $N^{*} f=\infty$ on a set of positive measure.

Definition 5.2. Let $(X, \beta, m)$ denote a measurable space and $\left\{R_{n}\right\}$ a sequence of positive operators defined on simple functions. Given $\delta>0$, $\left\{R_{n}\right\}$ is $\delta$-sweeping out if for all $\varepsilon>0$, there exists a measurable set $A$ with $m(A)<\varepsilon$ such that $\lim \sup _{n \rightarrow \infty} R_{n}\left(\chi_{A}\right) \geq \delta$ a.e.; and $\left\{R_{n}\right\}$ is strongly sweeping out if it is 1 -sweeping out.

COROllary 5.3. $\left\{B_{n}\right\}_{n}$ is $\delta$-sweeping out if and only if $\left\{N_{n} / n\right\}_{n}$ is $\delta$-sweeping out. 
Proof. This result is an immediate consequence of the relationship from Lemma 4.3,

$$
\frac{N_{n} \chi_{A}}{n}=B_{n} \chi_{A}
$$

EXAMPLE 5.4. Let $\tau$ be an ergodic measure preserving transformation on a probability space $(X, \beta, m)$. Let $\left\{n_{k}\right\}$ be a strongly sweeping out sequence, meaning that the averages along this subsequence, $n^{-1} \sum_{k=1}^{n} f\left(\tau^{n_{k}} x\right)$, form a sequence of strongly sweeping out operators. An example of such a sequence is $n_{k}=2^{k}$. See [2].

Now construct a sequence of measurable sets $A_{k}$ as follows.

Let $A_{k}$ be sets such that $m\left(A_{k}\right)<1 / 2^{k}$ and

$$
\limsup _{n \rightarrow \infty} \frac{N_{n}\left(\chi_{A_{k}}\right)}{n}=\limsup _{n \rightarrow \infty} B_{n}\left(\chi_{A_{k}}\right)=1 \quad \text { a.e. }
$$

Define $f=\sum_{k=1}^{\infty} k \chi_{A_{k}}$. Then $f \in L^{1}$. But since $f \geq k \chi_{A_{k}}$ for any $k \geq 1$, we have $N_{n}(f) \geq N_{n}\left(k \chi_{A_{k}}\right)$ for any $n \geq 1$, and

$$
\begin{aligned}
\limsup _{n \rightarrow \infty} \frac{N_{n} f}{n} & \geq \limsup _{n \rightarrow \infty} \frac{N_{n}\left(k \chi_{A_{k}}\right)}{n}=k \limsup _{n \rightarrow \infty} \frac{N_{k n}\left(\chi_{A_{k}}\right)}{k n} \\
& \geq \frac{k}{2} \limsup _{n \rightarrow \infty} \frac{N_{n}\left(\chi_{A_{k}}\right)}{n} \geq \frac{k}{2} \quad \text { a.e. }
\end{aligned}
$$

for any $k \geq 1$. Hence $N^{*} f=\infty$ a.e. as desired.

Consider now the operators

$$
S B_{p} f(x)=\left[\sum_{k=1}^{\infty} \frac{B_{k} f(x)^{p}}{k^{p}}\right]^{1 / p}
$$

and

$$
V_{p} f(x)=\left[\sum_{k=1}^{\infty}\left|B_{k} f(x)-B_{k-1} f(x)\right|^{p}\right]^{1 / p}
$$

where $B_{0} f=0$.

Let $S_{p} f(x)=\left[\sum_{k=1}^{\infty}\left|f\left(\tau^{n_{k}} x\right)\right|^{p} / k^{p}\right]^{1 / p}$.

LEMMA 5.5.

$$
S B_{p} f(x) \leq C(p) S_{p} f(x) .
$$

Hence $S B_{p}$ is a weak type $(1,1)$ operator and a bounded operator on $L^{\infty}$.

Before we prove this lemma, note that it does not follow from Corollary 5.11 because we are not dealing with blocks along the powers of one transformation. The proof in this one-dimensional case is quite simple and does not require the use of martingales as Theorem 5.12, used for Corollary 5.11, did. 
Proof. By the Hölder inequality,

$$
\left|B_{k} f(x)\right|^{p} \leq \frac{1}{k} \sum_{j=1}^{k}|f|^{p}\left(\tau^{n_{j}} x\right)=B_{k}\left(|f|^{p}\right)(x) .
$$

Thus,

$$
\begin{aligned}
S B_{p}^{p} f & =\sum_{k} \frac{\left|B_{k} f\right|^{p}}{k^{p}} \leq \sum_{k} \sum_{j=1}^{k} \frac{\left.\left|f\left(\tau^{n_{j}} x\right)\right|^{p}\right)}{k^{p+1}} \\
& \leq \sum_{j}\left|f\left(\tau^{n_{j}} x\right)\right|^{p} \sum_{k \geq j} \frac{1}{k^{p+1}}=C(p) \sum_{j} \frac{\left|f\left(\tau^{n_{j}} x\right)\right|^{p}}{j^{p}}=C(p) S_{p}^{p} f .
\end{aligned}
$$

Proposition 5.6. Let $1<p<\infty$. Then

$$
V_{p} f(x) \leq C\left[S_{p} f(x)+S B_{p} f(x)\right] .
$$

Hence, $V_{p} f$ is a weak type $(1,1)$ operator and is a bounded operator on $L^{\infty}$. Hence it is a strong type $(q, q)$ operator for $1<q<\infty$.

Proof. For $k>1$,

$$
B_{k} f(x)-B_{k-1} f(x)=\frac{f\left(\tau^{n_{k}} x\right)}{k-1}-\frac{B_{k} f(x)}{k-1} .
$$

Then

$$
V_{p}^{p} f(x) \leq 2^{p+1}\left[S_{p}^{p} f(x)+S B_{p}^{p} f(x)\right] .
$$

The rest of the proposition follows from Theorem 1.1 and Lemma 5.5.

REMARK 5.7. This result is interesting specially for sequences $\left\{n_{j}\right\}$ for which the averages $B_{k} f$ fail to converge. It shows that, even though the averages $B_{k} f$ may fail to converge, the difference of consecutive terms $B_{k} f-B_{k-1} f$ always goes to zero a.e. as $k \rightarrow \infty$.

Note 5.8. Both results 5.5 and 5.6 also hold without changes if one replaces the composition operators with $\tau^{n_{k}}$ by a sequence of operators $T_{k}$ satisfying

$$
\sum_{k=1}^{\infty} m\left(\left|T_{k} f(x)\right| \geq \lambda k\right) \leq \frac{C}{\lambda}\|f\|_{1} .
$$

5.2. Counting for $\mathbb{Z}_{+}^{d}$-actions. Let $\tau_{1}, \ldots, \tau_{d}$ be commuting measure preserving transformations of a probability space $(X, \beta, m)$. They define a $\mathbb{Z}_{+}^{d}$ semigroup action $\left\{\tau_{k}\right\}_{k \in \mathbb{Z}_{+}^{d}}$ by letting $\tau_{k} x=\tau_{1}^{k_{1}} \cdots \tau_{d}^{k_{d}} x$ for $k \in \mathbb{Z}_{+}^{d}$.

Let $\Gamma=\mathbb{Z}_{+}^{d}, \Gamma_{n}=[1, n]^{d}$, and

$$
\mathcal{S}_{n} f(x)=\sum_{k \in \Gamma_{n}} f\left(\tau_{k} x\right), \quad A_{n}^{d} f(x)=\mathcal{S}_{n} f(x) / n^{d} .
$$


Define now the counting function

$$
N_{\alpha} f(x)=\left|\left\{k \in \mathbb{Z}_{+}^{d}: \frac{\left|f\left(\tau_{k} x\right)\right|}{\|k\|_{\infty}^{d}}>\frac{1}{\alpha}\right\}\right|
$$

where $k=\left(k_{1}, \ldots, k_{d}\right)$ and $\|k\|_{\infty}=\max \left\{k_{1}, \ldots, k_{d}\right\}$. Define the maximal functions

$$
A^{*} f(x)=\sup _{n \geq 1}\left|A_{n} f(x)\right|, \quad N^{*} f(x)=\sup _{\alpha>0} \frac{N_{\alpha} f(x)}{\alpha} .
$$

In this situation we have operators $T_{k}$ induced by the point transformations $\tau_{k}$ : $T_{k} f(x)=f\left(\tau_{k} x\right)$. Let $g_{k}=\|k\|_{\infty}^{d}$, which gives $G_{n}=n^{d}$. It is easy to see that the $g_{k}$ 's are adapted to the operators $T_{k}$ 's with respect to the pair $(m, m)$, due to the integrability of the functions.

The index sets are $\Gamma_{n}=\left\{k \in \mathbb{Z}_{+}^{d}:\|k\|_{\infty} \leq n\right\}$. Then $\left\{T_{k}\right\}$ and $\left\{g_{k}\right\}$ satisfy the hypotheses of Theorem 4.4. Indeed,

$$
\begin{gathered}
\frac{G_{n+1}}{G_{n}}=\left[1+\frac{1}{n}\right]^{d} \leq 2^{d}, \\
\sum_{j \geq n} \frac{G_{j}-G_{j-1}}{G_{j}^{p}}=\sum_{j \geq n} \frac{j^{d}-(j-1)^{d}}{j^{d p}} \leq c(d) \frac{1}{n^{d(p-1)}}, \\
\left|\Gamma_{n}\right|=G_{n} .
\end{gathered}
$$

Since the operators $A_{n}^{d} f$ are the regular ergodic averages, $A^{*}$ is a weak type $(1,1)$ operator $([15])$. Thus Theorem 4.4 gives the following corollary.

Corollary 5.9. For any $p>1, N^{*}$ is a strong type $(p, p)$ operator.

Definition 5.10. A sequence $R_{n}$ of nondegenerate rectangles in $\mathbb{Z}_{+}^{d}$ is pseudo-increasing if (a) for each $n$ there exists $k_{n} \in \mathbb{Z}^{d}$ such that $R_{n}+k_{n}$ $\subset R_{n+1}$, and (b) $\left|R_{n}\right| \rightarrow \infty$ as $n \rightarrow \infty$.

This definition says that the rectangles are nested after a shift is performed on them.

Corollary 5.11. Let $T$ be a measure preserving action of $\mathbb{Z}_{+}^{d}$ on a probability space $(X, \beta, m)$. Define $A_{R_{n}} f(x)=\left|R_{n}\right|^{-1} \sum_{k \in R_{n}} f\left(T_{k} x\right)$ where $R_{n}$ is a pseudo-increasing sequence of nondegenerate rectangles in $\mathbb{Z}_{+}^{d}$. Then, for any $1<p<\infty$, the operator

$$
S_{p} f(x)=\left(\sum_{n=1}^{\infty} \frac{\left|A_{R_{n}} f(x)\right|^{p}}{n^{p}}\right)^{1 / p}
$$

is of weak type $(1,1)$. 
This corollary follows from Theorem 3.4 and the following result in Rosenblatt and Wierdl [14].

Theorem 5.12 (Rosenblatt \& Wierdl). Under the hypothesis of Corollary 5.11, there exists a constant $C(d)$ such that for any $f \in L^{1}$ and $\lambda>0$,

$$
\sum_{n=1}^{\infty} m\left(\left|A_{R_{n}} f\right|>\lambda n\right) \leq \frac{C(d)}{\lambda}\|f\|_{1} .
$$

The following result was already obtained for $p \geq 2$ and $d=1$ in [9], and for $d>1$ in [10]. Here is a simple proof for $1<p<\infty$ and $d \geq 1$.

Lemma 5.13. For any $1<p<\infty$, the operator

$$
V_{p} f(x)=\left(\sum_{n \geq 1}\left|A_{n}^{d} f(x)-A_{n+1}^{d} f(x)\right|^{p}\right)^{1 / p}
$$

is of weak type $(1,1)$ and bounded in $L^{\infty}$.

Proof. We have

$$
\begin{aligned}
\left|A_{n}^{d} f(x)-A_{n+1}^{d} f(x)\right| \leq & \left|\mathcal{S}_{n} f(x)\right|\left[\frac{1}{n^{d}}-\frac{1}{(n+1)^{d}}\right] \\
& +\left|\mathcal{S}_{n+1} f(x)-\mathcal{S}_{n} f(x)\right| \frac{1}{(n+1)^{d}} \\
\leq & c(d) \frac{\left|A_{n}^{d} f(x)\right|}{n}+\left|\mathcal{S}_{n+1} f(x)-\mathcal{S}_{n} f(x)\right| \frac{1}{(n+1)^{d}} .
\end{aligned}
$$

The first term is handled by Corollary 5.11 . The second term can be seen as the sum of $d$ averages of a $\mathbb{Z}_{+}^{d-1}$ action, divided by $n$. Indeed,

$$
\left|\mathcal{S}_{n+1} f(x)-\mathcal{S}_{n} f(x)\right| \leq \sum_{i=1}^{d} \mathcal{S}_{n+1}|f|\left(T_{i}^{n+1} x\right)
$$

where the $i$ th term of the sum corresponds to the $(n+1)$ th sum of a $\mathbb{Z}_{+}^{d-1}$ action:

$$
\mathcal{S}_{n+1}|f|(y)=\sum_{k \in[1, n+1]^{d-1}}|f|\left(\sigma_{1}^{k_{1}} \cdots \sigma_{d-1}^{k_{d-1}} y\right),
$$

with $\sigma_{j}=\tau_{j}$ if $j<i$ and $\sigma_{j}=\tau_{j+1}$ if $j>i$. Thus

$$
\frac{\left|\mathcal{S}_{n+1} f(x)-\mathcal{S}_{n} f(x)\right|}{(n+1)^{d}} \leq \sum_{i=1}^{d} \frac{A_{n+1}^{d-1}|f|\left(\tau_{i}^{n+1} x\right)}{n} .
$$

Let

$$
S_{p} f=\left[\sum_{n=1}^{\infty}\left(\frac{A_{n+1}^{d} f(x)}{n}\right)^{p}\right]^{1 / p}
$$


and for $1 \leq i \leq d$,

$$
S_{p}^{i} f=\left[\sum_{n=1}^{\infty}\left(\frac{A_{n+1}^{d-1} f\left(\tau_{i}^{n+1} x\right)}{n}\right)^{p}\right]^{1 / p} .
$$

By Corollary 5.11, $S_{p}$ and each $S_{p}^{i}, 1 \leq i \leq d$, is a weak type $(1,1)$ operator. Hence $V_{p}$ is also a weak type $(1,1)$ operator. Moreover, $S_{p}$ and the $S_{p}^{i}$ are also bounded in $L^{\infty}$, thus $V_{p}$ is also bounded in $L^{\infty}$.

5.3. Positive contractions in $L^{1}$. Let $(X, \beta, m)$ be a $\sigma$-finite measure space, and $U$ a linear operator defined on the measurable functions on $X$ satisfying

(a) $U$ is positive, that is, $U f \geq 0$ if $f \geq 0$,

(b) $U$ is an $L^{1}$ contraction.

Given $g \geq 0, g \in L^{1}$, and $f \in L^{1}$, define $\mathcal{S}_{n} g=\sum_{k=0}^{n-1} U^{k} g$ and

$$
N_{\alpha} f(x)=\left|\left\{k \geq 1: \frac{\left|U^{k} f(x)\right|}{\mathcal{S}_{k+1} g(x)} \geq \frac{1}{\alpha}\right\}\right|, \quad N^{*} f=\sup _{\alpha>0} \frac{N_{\alpha} f}{\alpha} .
$$

Lemmas 4.1 and 4.2 now apply with $\Gamma=\mathbb{Z}_{+}, g_{n}=G_{n}=\mathcal{S}_{n+1} g$ and $T_{n} f=U^{n} f$.

Let $m_{g}$ denote the measure induced by $g, m_{g}(A)=\int_{A} g d m$, and

$$
S_{p} f=\left[\sum_{k}\left(\frac{\left|U^{k} f\right|}{\mathcal{S}_{k+1} g}\right)^{p}\right]^{1 / p} .
$$

From the proof of the Chacon-Ornstein Theorem (Lemma 2.3 in [12]), we have the following estimate.

LEMMA 5.14.

$$
\sum_{k=1}^{\infty} m_{g}\left(U^{k} f(x)>\lambda \mathcal{S}_{k+1} g(x)\right) \leq \frac{\|f\|_{1}}{\lambda} .
$$

Theorem 3.4 and Lemma 5.14 now give the following square function result.

Corollary 5.15. For any $1<p<\infty$,

$$
m_{g}\left(S_{p} f>\lambda\right) \leq \frac{C}{\lambda} \int|f| d m
$$

Unfortunately, in this situation, the operators are not induced by measure preserving transformations, so we do not get the full strength of Theorem 4.4. Instead we obtain the following result.

Proposition 5.16. For any $1<p<\infty$ and $f \in L^{1}(m)$, the operator

$$
N_{p}^{*} f=\sup _{\alpha>0} \frac{\left(N_{\alpha} f\right)^{1 / p}}{\alpha}
$$


satisfies:

(a)

$$
N_{p}^{*} f \leq 2\left[\sup _{k} \frac{\left|U^{k} f\right|}{\mathcal{S}_{k+1} g}+1\right] S_{p} f
$$

almost everywhere on the set $[g>0]$,

(b) $N_{p}^{*}$ is a weak type $\left(L^{1}(m), L^{1}\left(m_{g}\right)\right)$ operator, that is, there exists a constant $C>0$ such that for any $f \in L^{1}(m)$ and $\lambda>0$,

$$
m_{g}\left(N_{p}^{*} f>\lambda\right) \leq \frac{C}{\lambda} \int|f| d m .
$$

Proof. First note that

$$
\sup _{\alpha \geq 1} \frac{\left(N_{\alpha} f\right)^{1 / p}}{\alpha} \leq 2 \sup _{n \geq 1} \frac{\left(N_{n} f\right)^{1 / p}}{n}
$$

and

$$
\frac{N_{n} f}{n^{p}} \leq \sum_{k=1}^{\infty}\left(\frac{\left|U^{k} f\right|}{\mathcal{S}_{k+1} g}\right)^{p}=S_{p}^{p} f
$$

For $\alpha<1$, let $\alpha_{0}=\alpha_{0}(x)$ be such that $1 / \alpha_{0}=2 \sup _{k}\left|U^{k} f(x)\right| / \mathcal{S}_{k+1} g(x)$. Then

$$
\sup _{0<\alpha \leq 1} \frac{N_{\alpha} f}{\alpha^{p}}=\sup _{\alpha_{0}<\alpha \leq 1} \frac{N_{\alpha} f}{\alpha^{p}} \leq \frac{1}{\alpha_{0}^{p}} N_{1} f \leq 2^{p} \sup _{k}\left(\frac{\left|U^{k} f\right|}{\mathcal{S}_{k+1} g}\right)^{p} \sum_{k=1}^{\infty}\left(\frac{\left|U^{k} f\right|}{\mathcal{S}_{k+1} g}\right)^{p} .
$$

Hence

$$
N_{p}^{*} f \leq 2\left[\sup _{k} \frac{\left|U^{k} f\right|}{\mathcal{S}_{k+1} g}+1\right] S_{p} f
$$

Thus,

$$
\begin{aligned}
m_{g}\left(N_{p}^{*} f>4\right) & \leq m_{g}\left(\sup _{k} \frac{\left|U^{k} f\right|}{\mathcal{S}_{k+1} g} S_{p} f>1\right)+m_{g}\left(S_{p} f>1\right) \\
& \leq m_{g}\left(\sup _{k} \frac{\left|U^{k} f\right|}{\mathcal{S}_{k+1} g}>1\right)+2 m_{g}\left(S_{p} f>1\right) \\
& \leq \sum_{k} m_{g}\left(\left|U^{k} f\right| \geq \mathcal{S}_{k+1} g\right)+2 m_{g}\left(S_{p} f>1\right) \leq C \int|f| d m .
\end{aligned}
$$

The weak inequality then follows because the operators $N_{p}^{*}$ are homogeneous of degree 1.

Proposition 5.17. Let $B_{k} f=\mathcal{S}_{k} f / \mathcal{S}_{k} g$. Then for any $1<p<\infty$, both

$$
V_{p} f=\left[\sum_{k=1}^{\infty}\left|B_{k+1} f-B_{k} f\right|^{p}\right]^{1 / p}
$$


and

$$
S B_{p}^{p} f=\sum_{k=1}^{\infty}\left(\frac{U^{k} g}{\mathcal{S}_{k+1} g}\right)^{p}\left|B_{k} f\right|^{p}
$$

are weak type $\left(L^{1}(m), L^{1}\left(m_{g}\right)\right)$ operators.

Proof. To show that $S B_{p}$ satisfies a weak type inequality from $L^{1}(m)$ to $L^{1}\left(m_{g}\right)$, it suffices, by Theorem 3.4 , to show that

$$
\sum_{k} m_{g}\left(U^{k} g\left|\mathcal{S}_{k+1} f\right|>\mathcal{S}_{k+1}^{2} g\right) \leq c\|f\|_{1} .
$$

But since $U^{k} g \leq \mathcal{S}_{k+1} g$, we have

$$
m_{g}\left(U^{k} g\left|\mathcal{S}_{k+1} f\right|>\mathcal{S}_{k+1}^{2} g\right) \leq m_{g}\left(\left|\mathcal{S}_{k+1} f\right|>\mathcal{S}_{k+1} g\right)
$$

and the result follows by Lemma 5.14.

For the other operator, write

$$
B_{k+1} f-B_{k} f=\frac{U^{k} f}{\mathcal{S}_{k+1} g}-\frac{U^{k} g}{\mathcal{S}_{k+1} g} B_{k} f .
$$

Then

$$
V_{p}^{p} f \leq 2^{p}\left[S_{p}^{p} f+S B_{p}^{p} f\right] .
$$

The weak type inequality for $V_{p}$ now follows from those for $S_{p}$ and $S B_{p}$.

\section{References}

[1] M. Akcoglu, J. Baxter, A. Bellow and R. L. Jones, On restricted weak type $(1,1)$; the discrete case, Israel J. Math. 124 (2001), 285-297.

[2] M. Akcoglu, A. Bellow, R. L. Jones, V. Losert, K. Reinhold and M. Wierdl, The strong sweeping out property for lacunary sequences, Riemann sums, convolution powers, and related matters, Ergodic Theory Dynam. Systems 16 (1996), 207-253.

[3] J. M. Ash, Weak restricted and very restricted operators on $L^{2}$, Trans. Amer. Math. Soc. 281 (1984), 675-689.

[4] I. Assani, Strong laws for weighted sums of independent identically distributed random variables, Duke Math. J. 88 (1997), 217-246.

[5] C. Bennett and R. Sharpley, Interpolation of Operators, Academic Press, 1988.

[6] A. Garsia, Topics in Almost Everywhere Convergence, Markham, Chicago, 1970.

[7] B. Jamison, S. Orey and W. Pruitt, Convergence of weighted averages of independent random variables, Z. Wahrsch. Verw. Gebiete 4 (1965), 40-44.

[8] R. L. Jones, Inequalities for the ergodic maximal function, Studia Math. 40 (1977), 111-129.

[9] R. L. Jones, R. Kaufman, J. Rosenblatt and M. Wierdl, Oscillation in ergodic theory, Ergodic Theory Dynam. Systems 18 (1998), 889-935.

[10] R. L. Jones, J. Rosenblatt and M. Wierdl, Oscillation in Ergodic Theory: Higher Dimensional Results, Israel J. Math. 135 (2003), 1-27.

[11] —, - - , Counting in ergodic theory, Canad. J. Math. 51 (1999), 996-1019.

[12] U. Krengel, Ergodic Theorems, de Gruyter Stud. Math. 6, de Gruyter, Berlin, 1985. 
[13] K. H. Moon, On restricted weak type $(1,1)$, Proc. Amer. Math. Soc. 42 (1974), $148-152$.

[14] J. Rosenblatt and M. Wierdl, A new maximal inequality and its applications, Ergodic Theory Dynam. Systems 12 (1992), 509-558.

[15] A. A. Tempel'man, Ergodic theorems for general dynamical systems, Dokl. Akad. Nauk SSSR 176 (1967), 790-793; English transl.: Soviet Math. Dokl. 8 (1967), 1213-1216.

[16] A. Torchinsky, Real-Variable Methods in Harmonic Analysis, Academic Press, 1986.

Department of Mathematics

University at Albany, SUNY

1400 Washington Ave.

Albany, NY 12222, U.S.A.

E-mail: reinhold@math.albany.edu

Received June 12, 2000

Revised version August 3, 2005 